\title{
The Reasons of Unequal Rainfall Distribution On The Earth And Its Forecasting Model In Azerbaijan
}

\author{
Mammadov Asgar Samed ${ }^{1}$, Rajabov Rustam Fakhraddin ${ }^{2}$, \\ Maharramova Malahat ${ }^{1}$ \\ ${ }^{\text {I} B a k u}$ State University, Faculty of Geography, Baku, Azerbaijan \\ ${ }^{2}$ State Water Resources Agency within the Ministry of Emergency Situation, Baku, Azerbaijan
}

\begin{abstract}
Two main aims are focused in the carried out article: 1) to clear out changable model of rainfall on the Earth by approaching with new form; 2) to work out rainfall forecast model. In relation with the first issue the results got from general circulation of atmosphere were analysed. Thus, influencing forces to atmosphere, in changing its rotary motion with regard to the Earth and the leading role of baric gradient force are explained. In the second issue, rainfall data line is expressed by harmonic function, periodic part is separated from the chance part and calculation are carried out on the base of analitic expression.
\end{abstract}

Keywords: rainfall, synoptic processes, harmonic analysis, solar activity, coriolis forces, friction force.

\section{Preface}

Distribution structure of rainfall according to place and time on the Earth is quiet complex. In some regions rain fall everyday, while in other there is no rain. Sometimes for some years rain falls very little or even no rain falls in the areas where, enough rain fell. Of course, a lot of researches were carried out in rainfall distribution field, but physical reasons of rainfall distribution differences have not been cleared out yet.

In 1934 Meynardus published average annual rainfall data according to geographical width. The quantities that he got were quiet different from the ones that Bryukner, Frich and Byust got. The rainfall maps that they worked out on the base of Shotta's data allow us to say that the distribution over the oceans are more complex and has positive correlation with general atmosphere circulation. As it is seen, a lot of rain falls in the regions around the equator and it lessens both to north and south. Its physical meaning is in formation of low pressure area in the equator. High pressure area formed in suptropical zone changes force correlation in atmosphere, and it serves as a background of rainfall decrease in these width.

As it is known, rainfall is one of the main indicators of the drought. That's why, rainfall forecast gives us opportunity to minimise drought consequences which is so dangerous for agriculture (Dai, A. 2013, Griffin, D., Anchukaitis, K. J. 2014) .

\section{The reasons of unequal rainfall distribution on the Earth}

As it is known, atmosphere movement happens as a result of rotary movement of the Earth. But the influencing forces on the atmosphere, effect its movement with the regard to the Earth. By the influence of these forces rotary motion of atmosphere with regard to the Earth increases and decreases, thus, when the direction of baric gradient force in the atmosphere is in the same way as its movement direction atmosphere motion is bigger than rotary motion of the Earth, otherwise, the rotary motion of the Earth is bigger than that of the atmosphere. Just, as a result of this movement, on the Earth surface in down width east wind blows, but in temperate and upper width west wind blows. So, atmosphere motion with regard to the Earth is late in down width, while in temperate and upper width, in contrary, atmosphere movemnet can be a bit higher than the Earth's one. Impulse moment for east wind is negative, but for west wind is positive. When the impulse moment gets higher, ie, west wind becomes stronger, rotary moment of the Earth decreases, and in this case rotary motion of the Earth weakens. When impulse moment of the atmosphere weakens, east wind becomes stronger (west weakens), rotary motion of the Earth increases (Sidorenkov N.S. 1982). According to the nature of influencing forces and preservation law of movement quantity it can be defined out that, atmospehere movement with regard to the Earth (depending on rate and direction of Baric gradient force) can be both late and early. But in free atmosphere it is late.

Atmosphere movement with regard to the Earth can happen under the influence of four forces: centrifugal force; friction force; Baric gradient and Coriolis forces. As centrifugal and friction forces are in higher altitude than border layer their influence is not taken into account. The rate of centrifugal force in the Earth surface is constant, friction force depends on wind speed (with regard of atmosphere to the Earth), Coriolis force depends on geographical width and relative movement speed. It must be taken into consideration one more thing that, the rate of friction force is more in dry surface than in water surface. That's why, as 
orographic characteristics become more complex in upper width the rate of friction force increases, with relation to this, in upper width (on ground surface) atmosphere movement brakes more.

Baric gradient force is the main accelarating force to air mass. This force plays important role in the formation of atmosphere processes. Just by the influence of this force the wind direction often changes, cloud and rainfall move from one part of the Earth to the other one. The rate of Baric gradient force depends on orographic characteristics, is componsated by friction and Coriolis forces. The unequal rainfall distribution on the Earth is mainly related with the change of Baric gradient force. Rate change of this force is defined according to temperature contrast between upper and down width. At high rate of temperature contrast the influence of advective processes increases in the regions, otherwise, ie, at low contrast rate convective processes weaken. Just as the temperature contrast often changes, the direction of Baric gradient vector changes too, as a result the movement of atmosphere rainfall changes over time. Surface cover of the regions around equator is more homogeneous than other width, nearly $20 \%$ of the area is dry and the rest is superficial. Here, the rate of friction force is a little changable, and is weaker in upper width, Coriolis force is considerable little. The homogeneity of the surface influences the change of Baric gradient force very little, decreases it. The reasons that rain falls too much to these width are more moisture and Baric gardient force is changable very little. That's why the rain falling to these regions is mainly convective charactered. The amount of rain falling in a narrow zone around equator during a year is $1500-2000 \mathrm{~mm}$. The rain falls a little less than $1000 \mathrm{~mm}$ from these regions to eastern costs of Africa, about $3000 \mathrm{~mm}$ rain falls in Borneo and Sumatra islands, upper parts of the Amazon river during a year. Formed differences in rainfall distribution in separate regions depend on orographic characteristics of the region. In general, the differences in rainfall distribution over space in the regions around equator is less than in other width (Marshall J. R. 1972).

The influence of Coriolis and friction forces towards upper width increases, the influence of Baric gradient force and rotary movement of the atmosphere with regard to the Earth changes more. That's why the amount of rain falling in western costal regions is less than the eastern one. Although Sahra desert stretches till the cost of the Atlantic ocean in Northern hemisphere the rain falling here is very little, no more than $250 \mathrm{~mm}$. The area in the western part of Northern America, especially from California peninsula towards north differs because of little rain. There is no such dry place in the east of Northern America. Generally, according to common accepted rules for passat zones, the rain falling to western part is less than the one in the eastern part. The situation quiet changes in temperate zones, western costs is more humid than eastern one. The rain increases till $2000 \mathrm{~mm}$ from $32^{0}$ width equator towards south in Southern America costs. Truely, rainfall is observed only in a very little zone, western slopes of the And mountains, then it decreases sharply, less than $200 \mathrm{~mm}$ a year (Marshall J. R. 1972).

Thus, one of the unequal rainfall distribution reasons over time and space is speed and direction change of atmosphere with regard to the Earth. So, the movement of atmosphere in the middle width differs from upper width by its speed and direction. In addition it must be noted that, the movement in upper layers from border stratum of atmosphere takes place upon geostrafic rules, ie, is defined by mutual influence of Baric gradient and Coriolis forces.

Atmosphere is less inert and more homogeneous in comparison with the Earth. That's why, the direction of movement speed of atmosphere rarely corresponds to the direction of rotary motion of the Earth and continous very short time.

\section{Annaul rainfall distribution in Azerbaijan}

Azerbaijan, like other parts of the world often undergoes rainfall difference. It must be noted that, the amount of average annual rainfall in the republic is about $496 \mathrm{~mm}$. But as the area owns complex orographic characteristics, it doesn't allow us to accept this calculation. Thus, as rainfall is too changable and ceasable over time and space it doesn't allow us to accept this calculation suitable for mountainous area. 450-500 $\mathrm{mm}$ rain falls in north-eastern slopes of the Great Caucasus, while the amount of rainfall in southern slope reaches to $1100 \mathrm{~mm}$ (Figure 1). The rain fell in September and October 2016 in Baku is approximately 5 times more than annual norm. That's why, according to the rainfall distribution characters the area is divided into 5 zones: coastal zone rainfall changes between $200-300 \mathrm{~mm}$; plain zone rainfall changes between $300-400 \mathrm{~mm}$; foothills zone rainfall changes between $400-600 \mathrm{~mm}$; middle mountainous zone rainfall changes between $600-800 \mathrm{~mm}$; high mountainous zone rainfall changes between $800-1300 \mathrm{~mm}$; and rainfall wavering involved in each type is defined separately.

The amount of rainfall for Baku, as a coastal region type, for the first 50 years decreased below norm, next 35 years increased, from the beginning of 1980 again decreased $80-90 \mathrm{~mm}$. From beginnig of those years (1980), it was defined that, the rainfall in Lankaran decreased till $200 \mathrm{~mm}$ (Mammadov, Rajabov, 2014).

The similar situation was observed for Guba region, too. Thus, in all areas mentioned above, in the first 50 years of the above noted period the amount of rainfall decreased about $100 \mathrm{~mm}$, next 50 years changed relatively less (decreased nearly $50 \mathrm{~mm}$ ), finally last 20 years decreased about $100 \mathrm{~mm}$. Of course, some 
abnormal years are excluded (Figure 1). Partial rainfall decrease for other regions of the country has been noted, too. In late 30 years a bit increase was observed in high mountainous zones.

Studying rainfall is too actual for Azerbaijan, because the area situates in arid zone characterized by 50 $\%$ semi-desert and dry steppe climate type. The analyse of data for 1881-2007 for Ganja and Kurdamir, situating in those area shows that rainfall decreased in those areas.

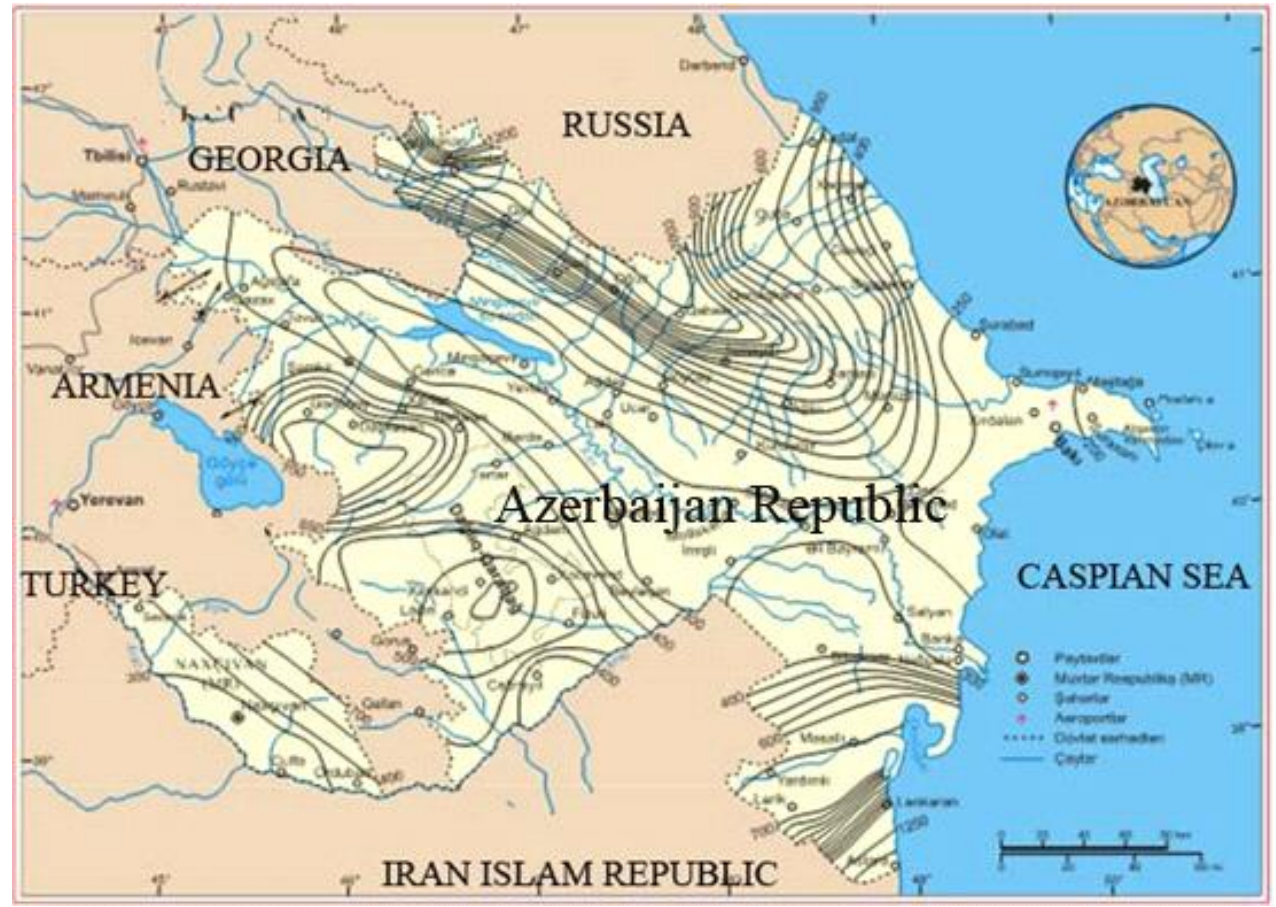

Figure 1. Average annual rainfall distribution in Azerbaijan.

In general, it was defined that rainfall decreased about $10 \%$ in late 30 years in the republic. It is known that, rainfall is a drought indicator. That's why, forecasting rainfall distribution over time is very important for minimisng expecting drought sedition. It must be noted that, although researchers strived too much in these fields, no success was got neither in drought, nor in rainfall forecast. But for some Azerbaijan regions definite forecast model for this field was proposed (Mammadov, Rajabov, 2014). Below, I direct your attention to one of these models.

\section{Rainfall forecast model}

Each process taking place in nature is divided into stationary and casual items. Revealing stationary effects forming process is easier than casual one. For example, by averaging long term data line which the process happened we can find out casual part. Thus, repeating orders (periodics) of stationary part are defined and forecast is prepared upon those stationary orders.

As it is known, meteorological observation data line can be divided into harmonic line with definite exactness. But, according to its length the period of this data line can be not equal to the length of harmonic. At this time, defining the periodics is carrried out upon different researches.

The following analytical expression is used for defining the existance of periodics (Brooks, Carruthers 1953) :

$y=A_{1} \sin \left(\frac{2 \pi}{\tau_{1}} t+\phi_{1}\right)$

Here:

$A_{1}=\sqrt{a^{2}+b^{2}} ; \varphi_{1}=\operatorname{arctg} \frac{a}{b} ; \quad a=\frac{2}{\tau_{c}} \sum_{i=1}^{\tau_{c}} y \cos \frac{2}{\tau_{c}} t ; \quad b=\frac{2}{\tau_{c}} \sum_{i=1}^{\tau_{c}} y \sin \frac{2 \pi}{\tau_{c}} t$.

It is known that, there are functions with different periods in each line structure. That's why, the analytic expression of that line can be written as following:

$y=A_{0}+A_{1} \sin \left(\frac{2 \pi}{\tau_{1}} t+\varphi_{1}\right)+A_{2} \sin \left(\frac{2 \pi}{\tau_{2}} t+\varphi_{2}\right)+\ldots .+A_{n} \sin \left(\frac{2 \pi}{\tau_{n}} t+\varphi_{n}\right)+\ldots+\theta$ 
Here $\theta-$ is a casual part in the given line.

Just, the evaluation of this item is in focus in the carried out work. At first, on the base of last function all items are defined, then by using solar activity data casual part is defined. In this case, numbers of 11 years' Solar cycle expressed by per cent are used.

\section{Calculations}

On the base of method given above, from inside the data of 1981-2009 in Azerbaijan nearly 20 periodics consisting of different period were defined (Mammadov, Rajabov, 2014). I think it is necessary to call your attention to some of them.

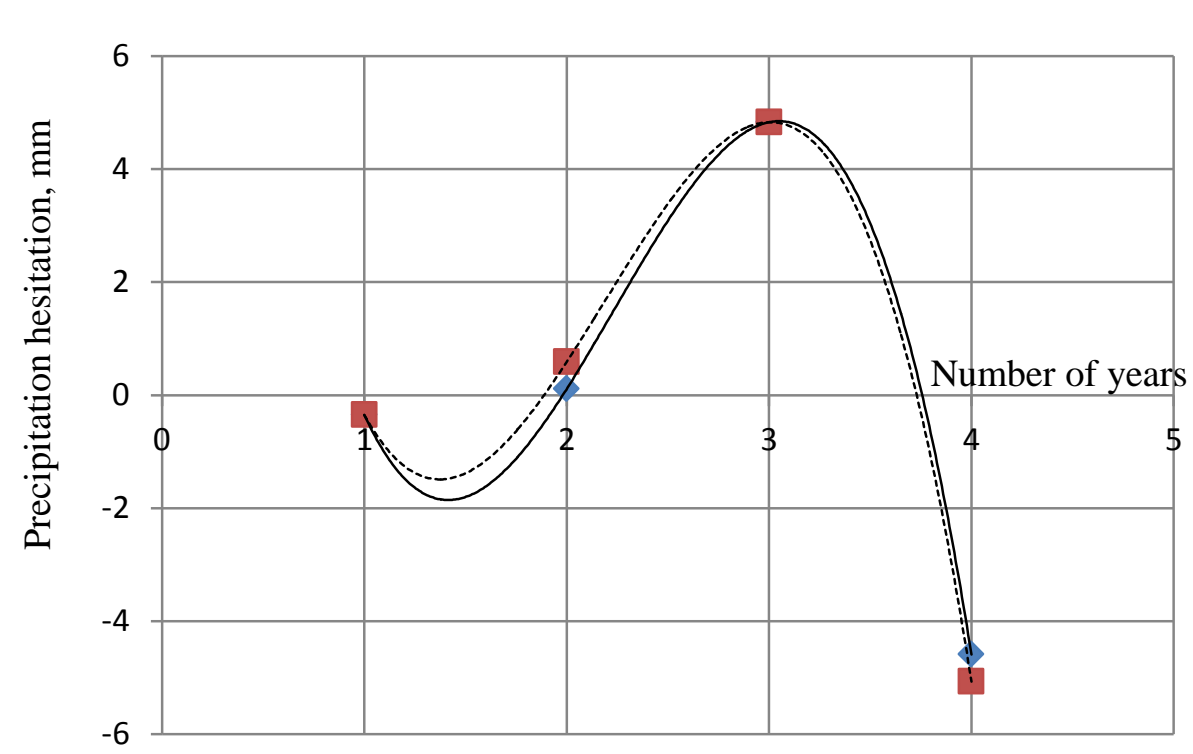

Figure 2. 4-year periodics in rainfall distribution. Blue coloured dots show calculations, red coloured dots show actual data.

4-year periodic is given in Figure 2, as it is seen calculation data and observation data correspond $100 \%$.

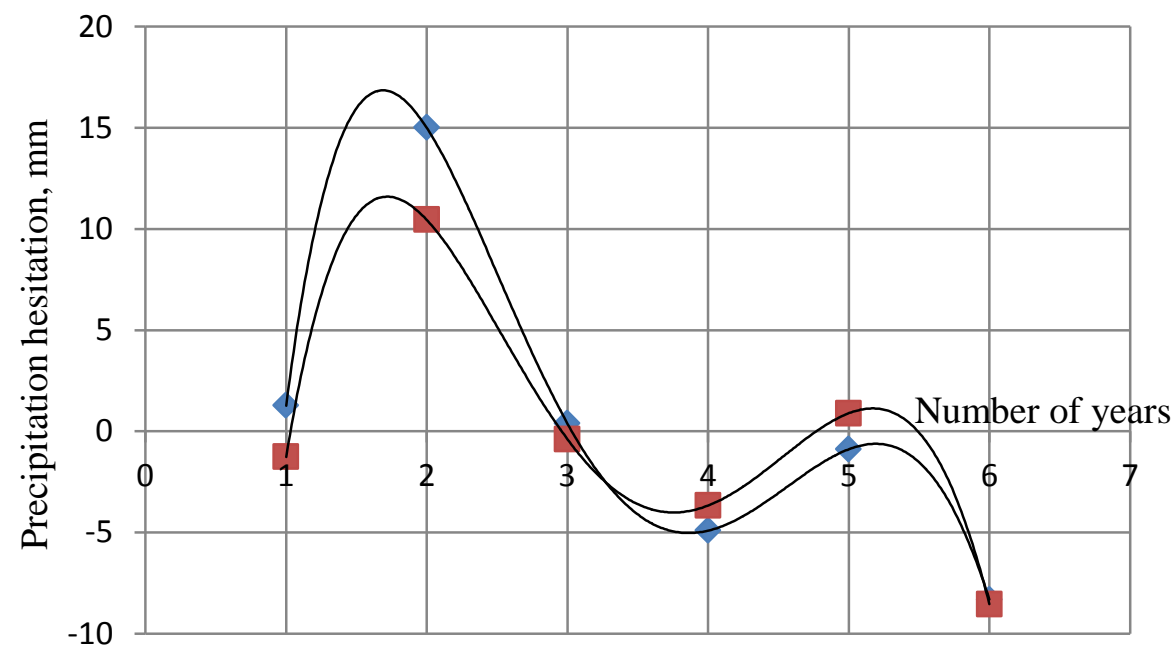

Figure 3. 6-year periodics in rainfall distribution. Blue coloured dots show calculations, red coloured dots show actual data. 


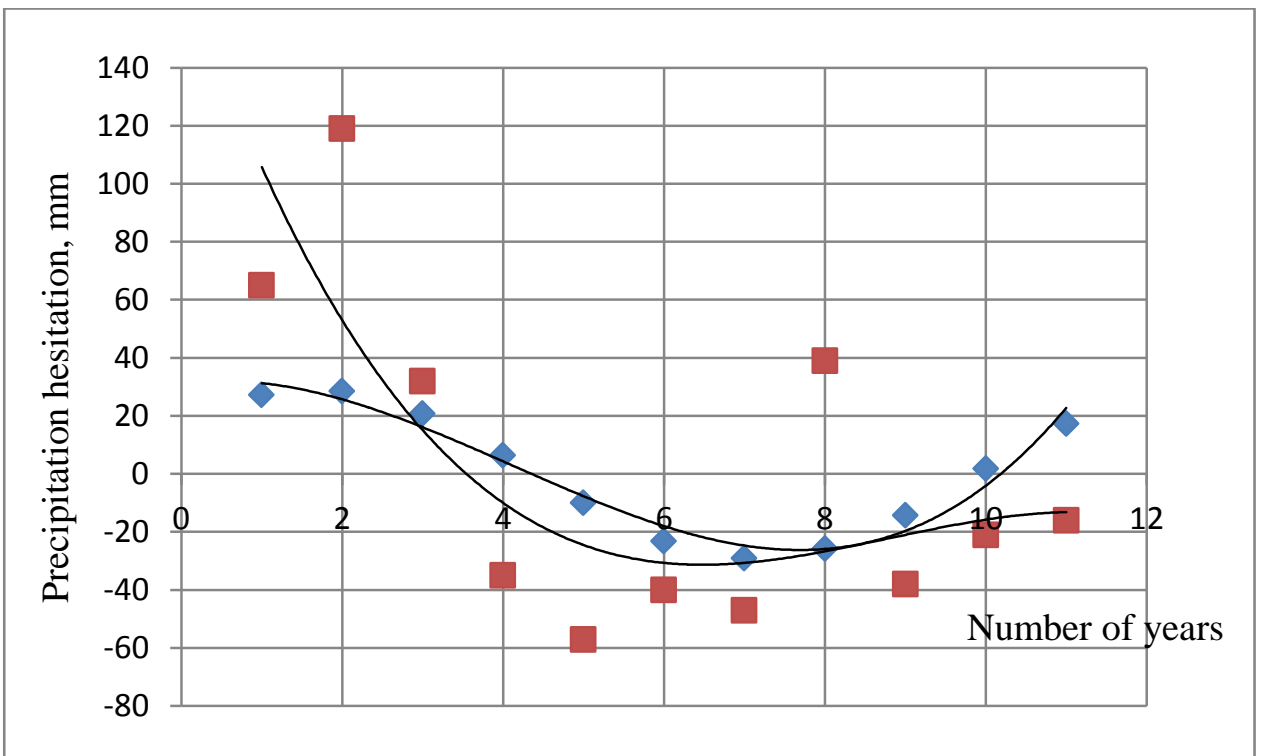

Figure 4. 11-year periodics in rainfall distribution. Blue coloured dots show calculations, red coloured dots show actual data.

Periodics defined for 6 - dots give us the basis to say that calculations completely correspond to initial data (Figure 3). 11-year periodics are shown in Figure 4. Though the repetition inside the Solar cycle is not convincing. So, the reachment of alarm happening in the Sun must be a bit late. In fact, the repetition happening in Solar cycle can continue 8-9 years. That's why, there is no place for doubts formed by 11 year periodic.

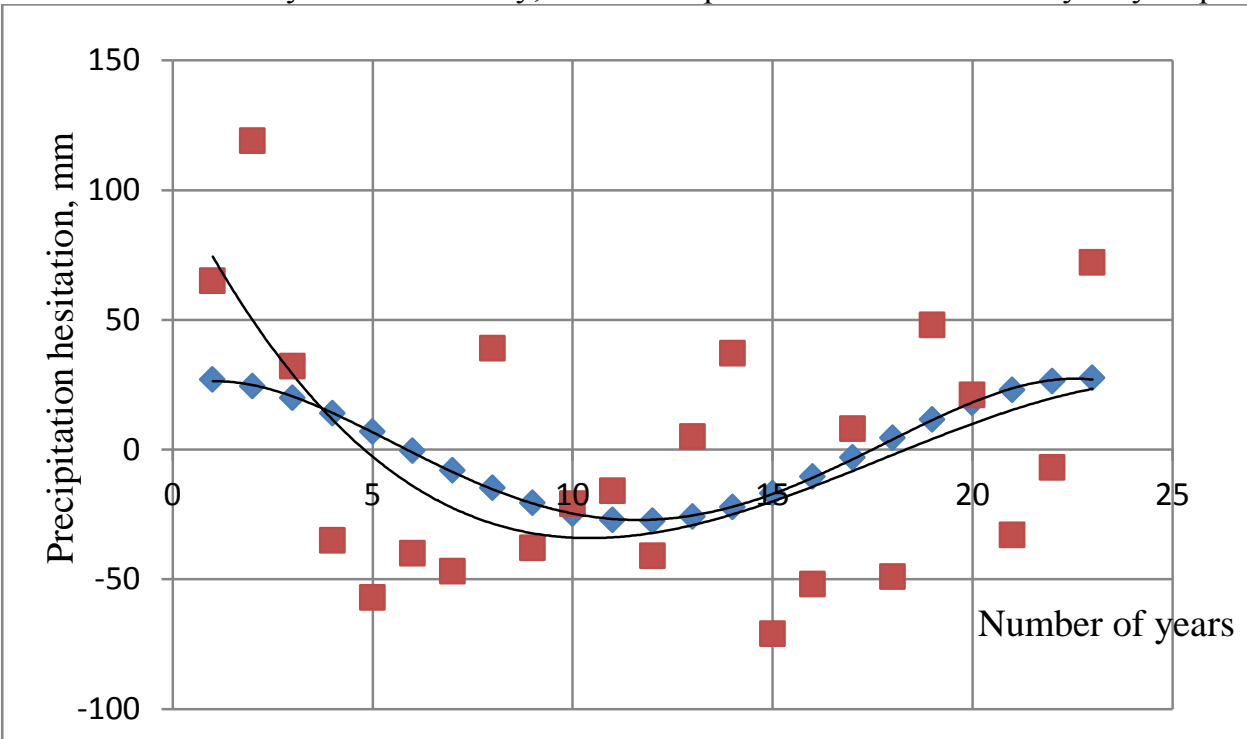

Figure 5. 24-year periodics in rainfall distribution. Blue coloured dots show calculations, red coloured dots show actual data.

A bit disorderliness of actual data in 24- year periodic does not show disparity with regard to calculation data, in contrary, it gives us the base to say that scattered data corresponds to symmetric calculation (Figure 5).

In Figure 6 on the base of observation station data on 1981-2009 (about 40) rainfall forecast is given till 2038. At first, if we pay attention to comparison of calculation data and actual data of 1981-2009, we can say that according to actual distribution the decrease beginning from 1981 (rainfall rate from $100 \mathrm{~mm}$ ) continued till 1988 (till $150 \mathrm{~mm}$ ), then there was no strong change in rainfall distribution, it changed around norm till 2000. In next phase after 2000 rainfall increased till $120 \mathrm{~mm}$, from beginning of 2005 rainfall again decreased till 100 $\mathrm{mm}$ with regard to the norm. By quality, annual move of calculation data takes place completely correspondent to actual data, the difference is only in quantity indicators. For example, the decrease beginning from $100 \mathrm{~mm}$ in actual data in 1981, began from $250 \mathrm{~mm}$ in calculations, the difference between second and third phase is no more than $50 \mathrm{~mm}$. 


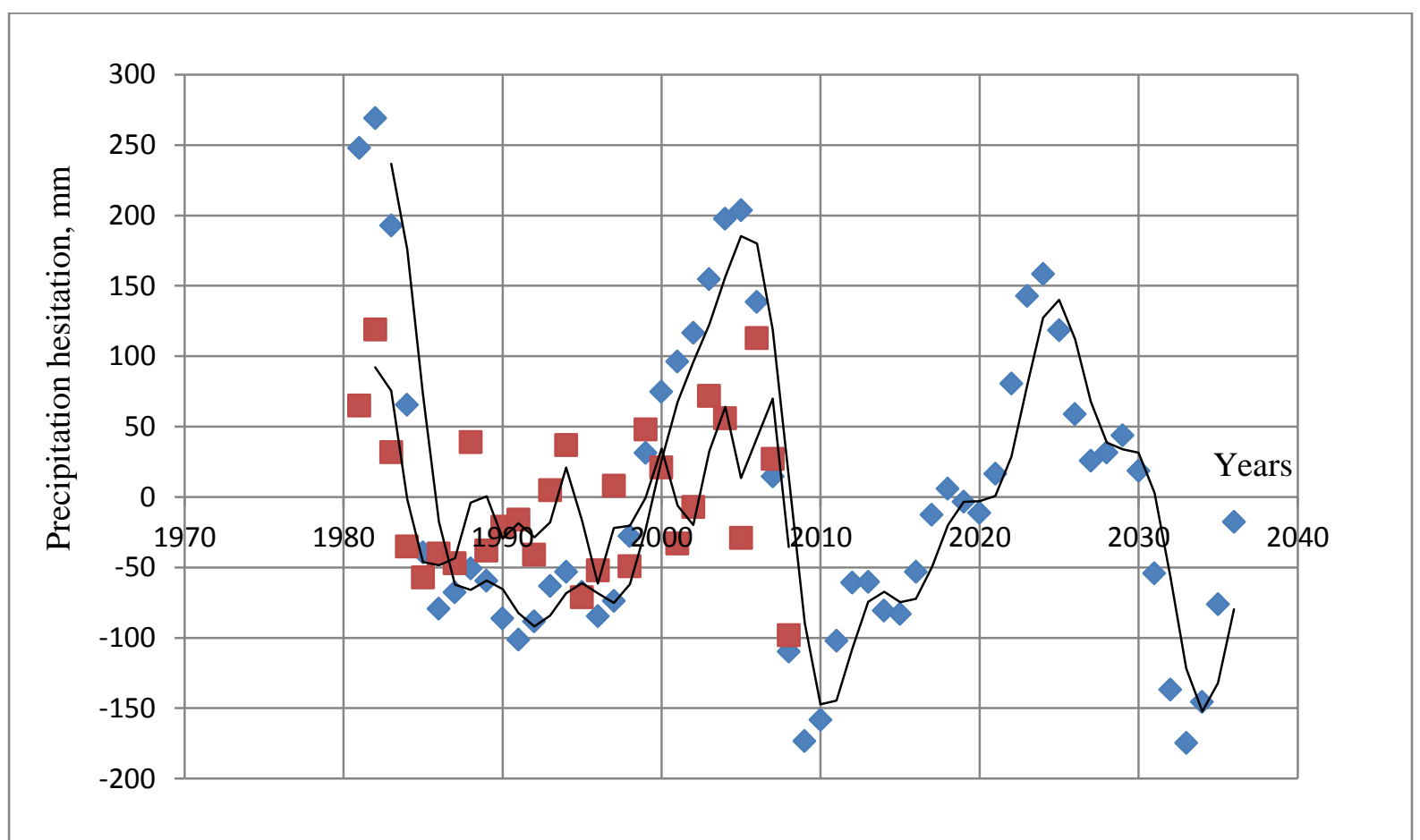

Figure 6. Calculation of rainfall distribution in Azerbaijan. Blue coloured dots show calculations, red coloured dots show actual data.

\section{Conclusion}

Rotary motion of atmosphere being different with regard to the Earth is shown as one of the new reasons of atmosphere rainfall distribution over time. The role of atmosphere force is defined in the change of these movement speeds. It is shown that atmosphere strata rotate with different speed in upper and middle width around the Earth. It was defined that Baric gradient force has exceptional role in atmosphere rainfall distribution.

The characteristic of rainfall distribution in Azerbaijan is cleared out on the base of designed rainfall map. The classification of rainfall distribution zones is given. Rainfall distribution forecast model is compiled, in this case approximately 20 periodics are found out. For the first time, for Azerbaijan area quality charactered rainfall forecast over time is given. According to the forecast, rainfall in Azerbaijan in 2020-2030 will be nearly $100 \mathrm{~mm}$ above norm, next 10 years will decrease $100 \mathrm{~mm}$. The accuracy of the given forecast is about $70 \%$.

Thus, if the rainfall is forecasted as about $100 \mathrm{~mm}$ below the norm, it must be understood that it will be a dry year, but if it is more, the year will not be dry.

\section{References}

[1]. BROOKS C. E. P., CARRUTHERS N, 1953. Handbook of Statistical Methods in Meteorology. Meteorological Office. Rpt. M. O. 538, H. M. S. O., London, pp: 331-365.

[2]. DAI, A. (2013). Increasing drought under global warming in observations and models. Nat. Clim. Change 3, 52-58.

[3]. GRIFFIN, D., ANCHUKAITIS, K. J. (2014). How unusual is the 2012-2014 California drought? Geophys. Res. Lett. 41, 2014 GL062433.

[4]. MAMMADOV, A.S., RAJABOV R F. (2015). Stochastic Model of Precipitation Prediction. International Jurnal of Scientific Research and innovative Technology. Vol. 2, No. 9; pp. 18 - 21 September.

[5]. MARSHALL J. R. (1972). Precipitation patterns of the United States and sunspots. PhD. Thesis, University of Kansas, Lawrense, Kansas.

[6]. SIDORENKOV N.S.(1982). Some parameters of the global water cycle according to the secular polar motion and the uneven rotation of the Earth//Water resources, № 3, p.39-46. 